\title{
Simultaneous mobile sink allocation in home environments with applications in mobile consumer robotics
}

Article

Accepted Version

Chanak, P., Banerjee, I. and Sherratt, R. S. (2015)

Simultaneous mobile sink allocation in home environments with applications in mobile consumer robotics. IEEE Transactions on Consumer Electronics, 61 (2). pp. 181-188. ISSN 0098-3063 doi:

https://doi.org/10.1109/TCE.2015.7150572 Available at https://centaur.reading.ac.uk/41207/

It is advisable to refer to the publisher's version if you intend to cite from the work. See Guidance on citing.

To link to this article DOI: http://dx.doi.org/10.1109/TCE.2015.7150572

Publisher: IEEE

All outputs in CentAUR are protected by Intellectual Property Rights law, including copyright law. Copyright and IPR is retained by the creators or other copyright holders. Terms and conditions for use of this material are defined in the End User Agreement. 


\section{CentAUR}

Central Archive at the University of Reading

Reading's research outputs online 


\title{
Full-Text version
}

Title: $\quad$ Simultaneous Mobile Sink Allocation in Home Environments with Applications
in Mobile Consumer Robotics

Authors: $\quad$ Prasenjit Chanak, Member, IEEE

Department of Information Technology, Indian Institute of Engineering Science and Technology, Shibpur, Howrah-711103, India, e-mail: prasenjit.chanak.2013@ieee.org

Indrajit Banerjee, Member, IEEE

Department of Information Technology, Indian Institute of Engineering Science and Technology, Shibpur, Howrah-711103, India, e-mail: ibanerjee@it.becs.ac.in

R. Simon Sherratt, Fellow, IEEE

School of Systems Engineering, University of Reading, Reading, Berkshire, RG6 6AY, UK, e-mail: sherratt@ieee.org

Publication: IEEE Transactions on Consumer Electronics

Volume: $\quad 61$

Issue: $\quad 2$

pp.: $\quad 181-188$

Date: $\quad$ May 2015

DOI: $\quad \underline{10.1109 / T C E .2015 .7150572}$

\begin{abstract}
This paper presents a novel mobile sink area allocation scheme for consumer based mobile robotic devices with a proven application to robotic vacuum cleaners. In the home or office environment, rooms are physically separated by walls and an automated robotic cleaner cannot make a decision about which room to move to and perform the cleaning task. Likewise, state of the art cleaning robots do not move to other rooms without direct human interference. In a smart home monitoring system, sensor nodes may be deployed to monitor each separate room.

In this work, a quad tree based data gathering scheme is proposed whereby the mobile sink physically moves through every room and logically links all separated sub-networks together. The proposed scheme sequentially collects data from the monitoring environment and transmits the information back to a base station. According to the sensor nodes information, the base station can command a cleaning robot to move to a specific location in the home environment. The quad tree based data gathering scheme minimizes the data gathering tour length and time through the efficient allocation of data gathering areas. A calculated shortest path data gathering tour can efficiently be allocated to the robotic cleaner to complete the cleaning task within a minimum time period. Simulation results show that the proposed scheme can effectively allocate and control the cleaning area to the robot vacuum cleaner without any direct interference from the consumer. The performance of the proposed scheme is then validated with a set of practical sequential data gathering tours in a typical office/home environment.
\end{abstract}

\section{Index Terms}

Mobile sinks, Wireless Sensor Network (WSN), Robotic Vacuum Cleaner, Clearing Area Assessment. 


\section{INTRODUCTION}

Recently, smart home network design has become an important research topic in order to provide consumers with the convenience of controlling smart home applications in the home [1]. Wireless Sensor Networks (WSNs) and Consumer Electronics (CE) technologies make a good combination to enable the design of smart home monitoring systems. To design a smart home network, sensor nodes are usually deployed into the home or office environment without a preconfigured infrastructure [2], [3]. Before monitoring the environment, sensor nodes are organized into a network through the neighbor nodes discovery process [4] to automatically gather real-time data from the home environment and control the operation of various digital household CE devices such as digital audio, entertainment, and air conditioning, etc. Nowadays, consumers can benefit from automatic selfcontrolled home aids including robotic vacuum cleaners that can automatically manage and control the movement of the vacuum cleaner pervasively within a home environment [5]-[9]. A smart home monitoring system can be applied to enable automatic maneuvering robotic vacuum cleaners within the home environment. WSNs are a promising technology for gathering real-time information in order to enable automatic monitoring of a specific area or to control electronic devices based on home environment conditions [10].

To design a WSN based smart home network, static sensor nodes are typically deployed to monitor separate rooms [2]. In each room, sensor nodes are deployed and connected, whereas sensors that belong to different rooms may be separated due to presence of walls. Without fully connected networks, a cleaning robot cannot make a decision about which room to move to and perform the cleaning task. As a result, some rooms typically cannot be serviced by the robotic device during the cleaning tour. A mobile sink based data gathering scheme collects data from all static sensor nodes and logically connects all sub-networks. In a mobile sink based data gathering mechanism, the shortest data gathering tour can reduce the data gathering latency and minimize energy consumption of the static sensor nodes [3]. During the data gathering tour, a mobile sink transmits cleaning area information to the Base Station (BS). According to the static sensor information, the BS can effectively manage the robotic cleaner mobility and assign a cleaning area for the robotic vacuum cleaner.

This paper proposes a mobile sink based data gathering scheme to control a single or suite of robotic vacuum cleaners within the home environment. A mobile sink starts the data gathering tour periodically from the nearest location of the BS in the home or office environment. While traveling, it polls each static sensor node in its transmission range and directly collects data from the static sensor by single-hop communications. Finally, the mobile sink transports the data to the BS. In a cleaning tour, the BS commands the robotic vacuum cleaner to move to a specific room in the home environment based on the previously collected sensor information.

According to the BS commands, the robotic vacuum cleaner moves to the target room and performs the cleaning task. The proposed quad tree based data gathering algorithm is used to control the mobility of the sink and assign a target room to clean in a distributed fashion. The distributed data gathering scheme further reduces the control latency for the cleaning robots through efficiently maneuvering within the environment.

A testbed has been developed that includes the static nodes as well as mobile sink created for verifying the effectiveness and feasibility of the proposed scheme. The performance of the experiment control network was tested using real-life scenarios. The stability of the proposed system for long-life deployed cleaning robots was also verified.

The rest of the paper is organized as follows: in Section II a literature review on robotic vacuum cleaner path planning is presented; Section III gives the home network system; Section IV gives an analytical description of the proposed scheme; Section V presents experimental and analysis results and Section VI finally concludes the paper.

\section{RELATED WORKS}

Many studies have been performed to address different problems for automatic maneuvering of robots in the home environment.

Saeedi et al. [11] proposed a neural network based multiple robot localization and mapping scheme. This technique used a map learning method for localization of the multiple robots. They used self-organization maps 
to reduce the complexity of the acquired occupancy grid maps.

Doh et al. [5] proposed a path planning strategy for robotic vacuum cleaners movement in a rectilinear environment. They considered two major issues to design an effective mobility path; a) human-friendly path generation, and b) low computational load.

Kim et al. [1] proposed a vision based system for a robotic vacuum cleaner mobility management. It used a Human Computer Interaction (HCI) method to control the cleaning robot in order to direct it to a particular location in the environment.

Lee and Drysdale [12] proposed a generalized Voronoi graph (GVG) for path planning in topological environments. This technique exhibited a zigzag motion to gather sense information.

Choset and Burdick [13] proposed a sensor based motion planning technique for a mobile robot. It used a hierarchical generalized Voronic graph for motion planning.

Geraerts and Overmars [14] proposed a small roadmap to reduce the large number of nodes of the probabilistic roadmap. This roadmap decreased the node number by placing a node in a spot which the other nodes cannot see.

Despite the significance of the technical problems concerned with the simultaneous allocation of robotic vacuum cleaners, so far none of the existing solutions have provided an efficient method for the simultaneous allocation in typical home or office environments. The existing works are primarily based on an existing map or human computer interaction and none of them have considered the area overlapping problem in the path design process. In the following presentation, a dynamic tour planning strategy is shown that is based using real-time sensing in a realistic and efficient automatic maneuvering robotic vacuum cleaner in a typical home or office home environment. This is the main motivation and contributions from this work.

\section{SYSTEM MODEL}

Fig. 1 shows a smart home network model consisting of statically deployed sensors as well as mobile sink(s) which play an important role in this work. A cleaning robot equipped with a wireless transceiver is used as the mobile sink. Static sensors are deployed within the home or office environment where rooms are separated by walls, therefore the home network is physically divided into several sub-networks. A mobile sink starts a data gathering tour periodically from a known location in a known room and collects data from the static sensors while traversing its transmission range. The mobile sink finally transports the found data back to the BS. According to the static sensor information, the BS efficiently allocates a cleaning area to the robotic vacuum cleaner and automatically manages the robotic vacuum cleaner movement within the home environment. This work implements a quad tree based optimal data gathering tour planning mechanism whereby a mobile sink can visit all sub-networks or rooms through the optimal path and gather data from the static sensor nodes. The optimal data gathering path can therefore minimize the data collection time and maximize the lifetime of the smart control network. A quad tree based optimal multiple sink mapping strategy is also used that can control the movement of multiple sinks which effectively manages multiple vacuum cleaners within a large size home or office environment. 


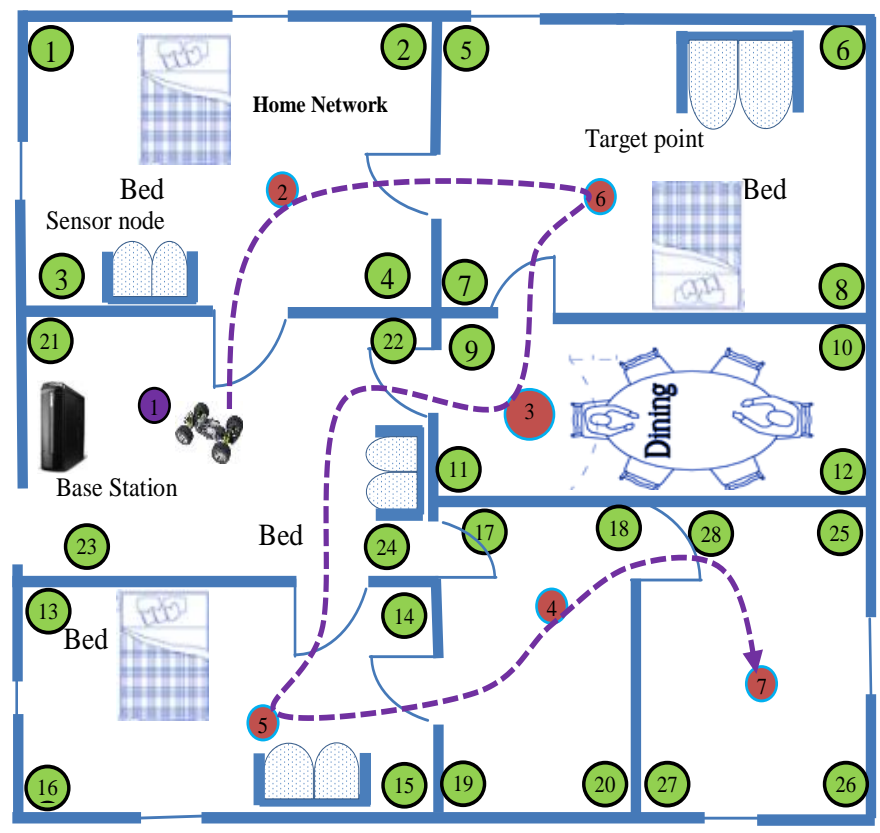

Fig. 1. System model.

The radio model for energy is used in this work to evaluate the performance of the smart home network [15]. In this model, energy consumption by a source node to transmit a $\beta$ bit long data message to a destination node is given as:

$$
E_{T x}= \begin{cases}\left(\alpha_{t x}+\varepsilon_{f s} d^{2}\right) \beta & d<d_{0} \\ \left(\alpha_{t x}+\varepsilon_{m p} d^{4}\right) \beta & d \geq d_{0}\end{cases}
$$

where, $\varepsilon_{f s}$ and $\varepsilon_{m p}$ are the energy required in free space and multipath communication respectfully. The $\alpha_{t x}$ represents one bit data transmitting energy loss. If the distance $(d)$ is less than a threshold value $d_{0}$, then the free space $(f s)$ model is used, otherwise, a multipath $(m p)$ model is considered.

The energy consumption by a destination node is given as:

$$
E_{R x}=\delta_{1} \beta
$$

where, $\delta_{l}$ represents the energy dissipation by the receiver circuit to receive one bit data.

\section{Cleaning Area Allocation Scheme in a Home EnVironment}

In this section, the quad tree formation and mobile sink based data gathering strategy is studied briefly followed by the robotic vacuum cleaner movement in a home environment. This section also describes the cleaning area allocation and maneuvering scheme for the cleaning robots.

\section{A. Quad-Tree Based Network Partitioning Strategy}

This proposed scheme splits the entire network into $\delta$ size regions with respect to the central nodes set, $C=$ $\left\{C_{l}, C_{2}, \ldots, C_{i-1}, C_{i}\right\}$. The $C_{l}$ node is a normal sensor node that can divide the entire network into different regions. Each region is supervised by a central node $C_{i}$, where $i$ is the highest level value. The $C_{i}$ node of a particular region is connected with a $C_{i-1}$ node of the upper region. The quad tree based network partitioning scheme logically divides the entire home monitoring network into different regions according to the room setup and allocates a mobile sink to a specific room or region by the distributed faction. A distributed mobile sink 
allocation strategy is perfectly suitable for a home application where rooms are separated by walls. It can reduce data gathering latency and improve performance of the network. The detailed description of the quad-tree based logical region partitioning process is discussed below:

1.Initially, the BS selects a $C_{l}$ node within the network. The x, y position of the $C_{l}$ node at $l \times \omega$ area is represented by N.XVAL and N.YVAL where:

$$
\begin{aligned}
& N . X V A L=\frac{1}{2 \times \text { level }} \\
& N . Y V A L=\frac{\omega}{2 \times \text { level }}
\end{aligned}
$$

where level is the present partition number.

2. The $C_{l}$ node logically divides the entire network into four regions, NW (northwest) region, SW (southwest) region, NE (northeast) region and SE (southeast) region. These four regions are then directly supervised by the $C_{l}$ node. The $C_{l}$ node controls the data gathering tour from the top level of the quad-tree. It effectively deals with the sink mobility in the home environment.

3. The $C_{1}$ node farther selects four $C_{2}$ nodes $\left\{C_{2 N W}, C_{2 S W}, C_{2 N E}, C_{2 S E}\right\}$ in the central position of each region. The $C_{2 N W}$ node position in NW region is:

$$
\begin{aligned}
& N . X V A L_{2 N W}=\frac{1}{2 \times \text { level }} \\
& N . Y V A L_{2 N W}=N . Y V A L+\frac{\omega}{2 \times \text { level }}
\end{aligned}
$$

Similarly, $C_{2 S W}, C_{2 N E}$ and $C_{2 S E}$ nodes' positions are selected at the middle position of each region. The $\left\{C_{2 N W}, C_{2 S W}, C_{2 N E}, C_{2 S E}\right\}$ node further divides each region into four sub regions NW region, $\mathrm{SW}$ region, NE region and $\mathrm{SE}$ region.

4. $C_{2 N W}, C_{2 S W}, C_{2 N E}, C_{2 S E}$ central nodes are the child node of an upper level $C_{1}$ node. In this way, the whole network is divided into several $\delta$ size regions. Each small region is directly supervised by one central node $C_{i}$ as shown in Fig. 2.

5.Central nodes generate a virtual quad tree for better network management and efficient cleaning area assessment of the robotic cleaner.

6.Each upper level $C_{j}$ node manages sink allocation process between its child $C_{j+1}$ nodes. However, bottom level $C_{i}$ node controls data gathering sub-tour under its regional nodes.

7.Parent $C_{j}$ node makes a sink allocation decision on the basis of child $C_{j+l}$ node information. In other words, child $C_{i}$ nodes do not interfere with the parent node decision making process.

8. While the mobile sink is travelling within a static sensor transmission range, it directly collects data from the static sensor node using single hop communication and transports the data up to the BS. 


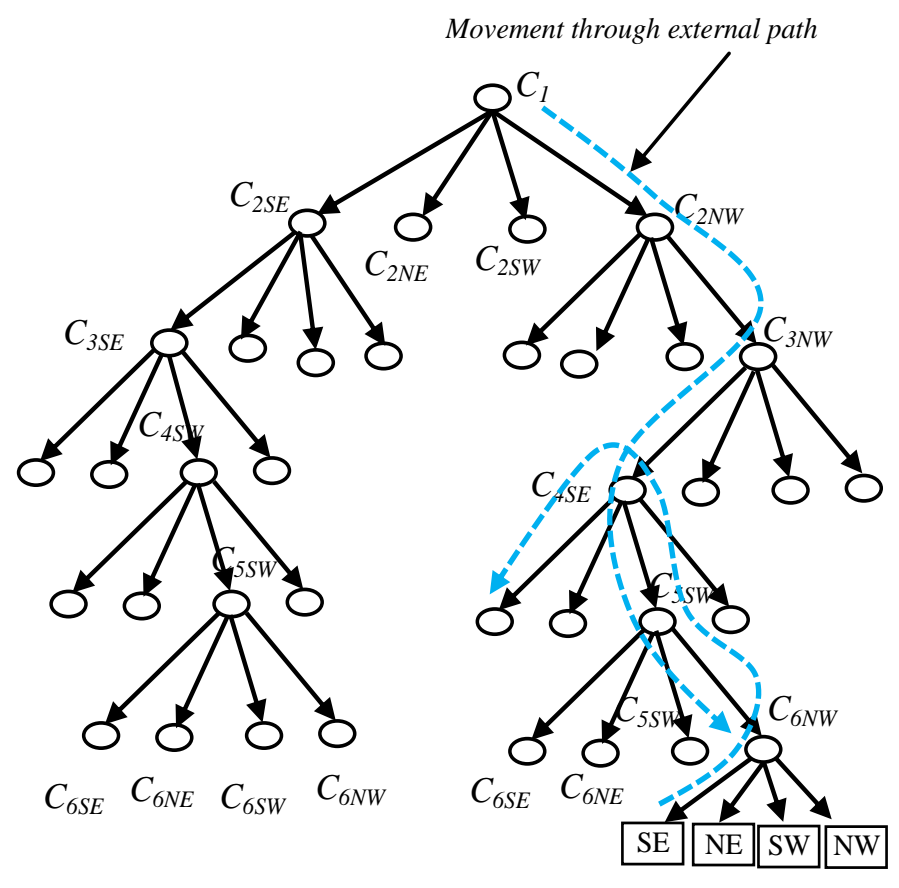

Fig. 2. Quad-tree relationship between the central nodes.

In a data gathering tour, the mobile sink follows three types of mobility paths in order to gather data from different rooms in the home environment. These three mobility paths are continually managed by the central nodes set and the BS respectively. Before, the data gathering schemes are defined, the three mobility paths that will be used in the rest of the paper need to be defined as:

1.External trip mobility path: the mobile sink follows external trip movement paths within the data gathering tour when it travels one level region to another level region within the quad tree.

2.Inner trip mobility path: the mobile sink follows internal trip movement paths within a data gathering tour while it travels one region to another region within the same level of the quad tree.

3. Homegrown mobility path: the mobile sink moves through the homegrown data collection paths when it travels within the region or sub-network in a data gathering tour.

The external trip and the inner trip mobility path of a data gathering tour is minimized by the quad tree based mobility management scheme. However, the homegrown path is minimized on basic neighbor node distance. The mobile sink starts data gathering tour data from the BS and at the end of the data gathering tour the mobile sink returns to the BS.

A data gathering tour is divided into different sub-tours, according to the quad information. Each optimum data gathering sub-tour effectively gathers data from each sub-network. The starting and ending points of a subtour is a lower level $C_{i}$ node.

\section{B. Maneuvering of the Mobile Sink in an External Trip Movement Path}

This section considers the optimum sink mobility problem for the external trip movement path. The external trip movement path starts from the upper level $C_{l}$ node and ends at the bottom level $C_{i}$ node within the quad tree. According to the availability of the child central nodes, the upper level central nodes allocate a lower level child central node to the mobile sink. For optimal sink mobility management, each level $C_{j}$ node manages four Sink Allocation Variables (SAV) for its four child central nodes. When a child $C_{j+1}$ node is allocated to a mobile sink by the parent $C_{j}$ node, then SAV is incremented by one by the parent $C_{j}$ node. The upper limit of the SAV is four which indicates the maximum four mobile sinks that can be allocated to a child $C_{j+1}$ node simultaneously by the 
parent node.

Fig. 2 illustrates the sink mobility management process in the external trip movement path. For example, let a mobile sink start the data gathering tour from the nearest area of the $C_{l}$ node by sending a "Allocate_Request" message to $C_{l}$ node. When $C_{l}$ receives the "Allocate_Request" message from the mobile sink, it identifies the lowest SAV child node from its $C_{2 N W}, C_{2 S W}, C_{2 N E}$, and $C_{2 S E}$ child node set and lowest SAV child node allocated to the mobile sink. If $C_{l}$ node detects the same $\mathrm{SAV}$ value of all its child nodes then node $C_{l}$ can start the allocation process using a right to left scanning process. In the initial condition of a data gathering tour, node $C_{l}$ allocates node $C_{2 S E}$ to the mobile sink by sending "ACK" to the mobile sink. When mobile sink reaches node $C_{2 S E}$ then $C_{2 S E}$ can allocate the next level central nodes to the mobile sink through the same allocation process. Throughout the allocation process when mobile sink reaches to the bottom level $C_{i}$ node, the mobile sink can start a data gathering sub-tour data within the region through homegrown data collection path. Then, external trip mobility path can be represented by:

$$
C_{1} \rightarrow C_{2 N W} \rightarrow C_{3 N W} \rightarrow C_{4 S E} \rightarrow C_{5 S W} \rightarrow C_{6 N W}
$$

In a data gathering tour, sink mobility time within the external trip mobility path is calculated by:

$$
T_{E x_{-} P a t h}=\sum_{1}^{n}\left(t_{\text {shift }}+t_{\text {allocation }}\right)
$$

where, $n$ is the total number of shifts between the different levels within the quad tree. The $t_{\text {shift }}$ represents travelling time of the mobile sink from one level to another level region, it depends on the speed of the mobile sink, $t_{\text {allocation }}$ represents the child node allocation time.

\section{Maneuvering of the Mobile Sink in an Internal Trip Movement Path}

A mobile sink follows the internal trip movement path when it moves from one sub-network to another subnetwork under the same $C_{i}$ node within the quad tree. During the shifting process, the mobile sink can communicate several times to the local $C_{i}$ node, as defined in Fig. 3, therefore, at this time, $C_{i}$ node moves to a hold state. When a $C_{i}$ node moves into the hold state it cannot accept any external requests by any other mobile sink without successful shifting the current mobile sink. After successful completion of the shifting process, $C_{i}$ receives new allocation requests via a First Come First Serve (FCFS) selection process from the requests queue. When a $C_{i}$ node allocates a new sub network to a mobile sink it then first checks the homegrown path condition. If all homegrown paths under the $C_{i}$ node are already allocated or visited by the any mobile sink, $C_{i}$ communicates to its parent $C_{i-1}$ node for new allocation. Then, the $C_{i-l}$ central node checks SAV values to allocate a new $C_{i}$ child node to the requesting mobile sink. After allocation of the new region by $C_{i-1}$ central node, the mobile sink follows an internal trip movement path for moving from one child $C_{i}$ node to the same level of another child $C_{i}$ node. However, if the $C_{i-1}$ node finds that all child nodes have already been allotted or visited by the mobile sink, it communicates to its $C_{i-2}$ parent node to allocate a new data gathering path to the requesting mobile sink. In this case, the $C_{i-2}$ node can allocate another $C_{i-1}$ node according to SAV values. Similarly, the $C_{i-1}$ node also finds an effective $C_{i}$ child node and allocate to the mobile sink. The child $C_{i}$ node allocates an unvisited sub-network to the requesting mobile sink and conducts a sub data gathering tour within the allocated region or room. In this shifting process, if a newly allocated region is present at the same level then the mobile sink also follows the internal trip movement path to move from one region to another region. However, if a newly allotted region is present at another level of the quad tree, the mobile sink uses the external trip movement for shifting process. For example, in Fig. 3, the mobile sink reaches the $C_{6 N W}$ node in a data gathering tour via the external trip movement path and it gathers data through the local data collection path from the all regions under the $C_{6 N W}$ node. After competition of the data gathering process, mobile sink communicates to $C_{6 S W}$ node for next region allocation and $C_{5 S W}$ node communicates to $C_{5 S W}$ node for new allotment. When $C_{5 S W}$ receives allotment request from the mobile sink then it verifies the target values of all child nodes $C_{6 S W}, C_{6 N E}$, and $C_{6 S E}$ and allocates a new $C_{6 S W}$ node for conducting another sub data gathering tour. After allocation of all child nodes under node $C_{5 N W}$, internal trip movement path within the data gathering tour can be represented by: 


$$
C_{5 S W} \rightarrow C_{6 N W} \rightarrow C_{6 S W} \rightarrow C_{6 N E} \rightarrow C_{6 S E} \rightarrow C_{5 S W}
$$

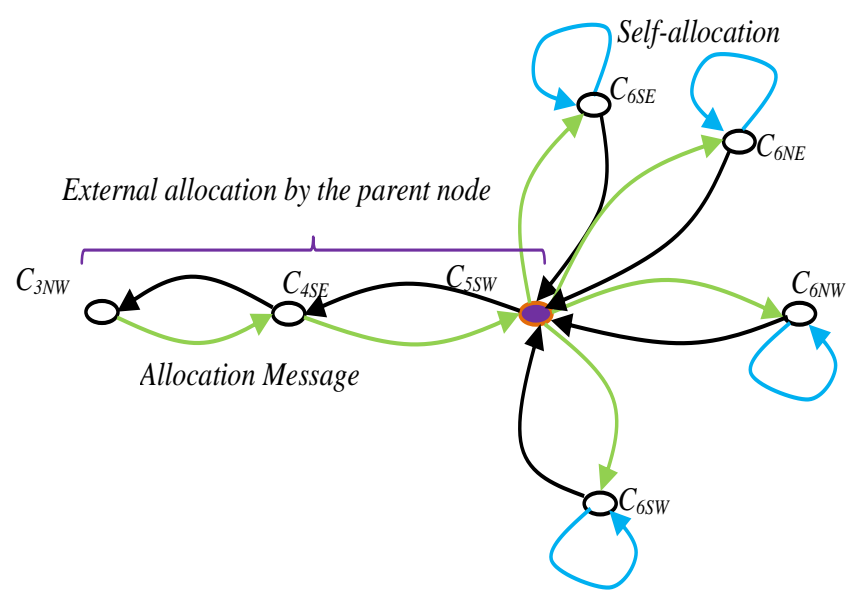

Fig. 3. Hold state condition.

In a data gathering tour, sink mobility time within the internal trip movement path is calculated by:

$$
T_{\text {Inter_Path }}=\frac{N+1}{4}\left(t_{\text {shift_time }}+t_{\text {allocation }}\right)
$$

where, $N$ is the number of central nodes and $t_{\text {shift_time }}$ is the shifting time of the mobile sink from one region to another region.

\section{Maneuvering of the Mobile Sink in a Homegrown Mobility Path}

The mobile sink follows the homegrown mobility path when it gathers data within the sub-network. A homegrown mobility path starts from the lower level $C_{i}$ node and after visiting all regional sensors it ends at $C_{i}$. Within the region, when a mobile sink reaches each static sensor it then collects data by single hop communication and it transmits back to the BS. Within the region, the mobile sink visits each static sensor node for data collection through the nearest neighbor node detection process.

For large scale home or office areas, each data gathering tour may take a long time so that a single mobile sink may not be sufficient to visit the all target rooms. A multiple mobile sink based data gathering scheme can be used to reduce data gathering time in a large scale home or office area. In a multiple mobile sink based data gathering scheme, multiple mobile sink management is one of the major challenging problems to solve. The next section will address the multiple mobile sink maneuvering problem.

\section{E. Maneuvering of Multiple Mobile Sinks}

In the multiple mobile sink based data gathering scheme, all central nodes of the quad tree concurrently manage sink mobility at each level. When a sink moves from one $C_{i}$ node to another $C_{i}$ node, then the $C_{i}$ node communicates to $C_{i-1}$ node for allocation of the new region. Each $C_{i}$ node discloses the SAV values for the sink allocation process. In this proposed scheme, each $C_{i}$ node can allocate a maximum of four numbers of sinks for a particular child node. Because each ground level $C_{i}$ node controls four sub-network or homegrown paths at any time, then only one mobile sink can gather data from a sub-network using the homegrown path. Central nodes follow mutual distribution and the FCFS strategy for sink distribution between the child nodes. The mutual sink distribution process distributes loads equally within all sinks and also reduces the data gathering latency. 


\section{F. Area Allocation and Maneuvering of Robots}

Fig. 4 shows the automatic cleaning area allocation scheme for the robotic cleaner. In the cleaning area allocation scheme, the BS uses the static sensor information that has been collected by the mobile sink(s). Initially, the BS assigns the nearest region to the robot and commands the robot to move to that assigned location or regions in the home environment according to the external trip movement path. After completion of the task within the allocated region, the robot informs the BS and the local $C_{i}$ node. The $\mathrm{BS}$ and $C_{i}$ node assigns the next nearest region to the robot according to the sensor information by a self-allocation or external allocation process. The cleaning robot then moves to the allotted area through the external trip mobility path or inner trip mobility path.

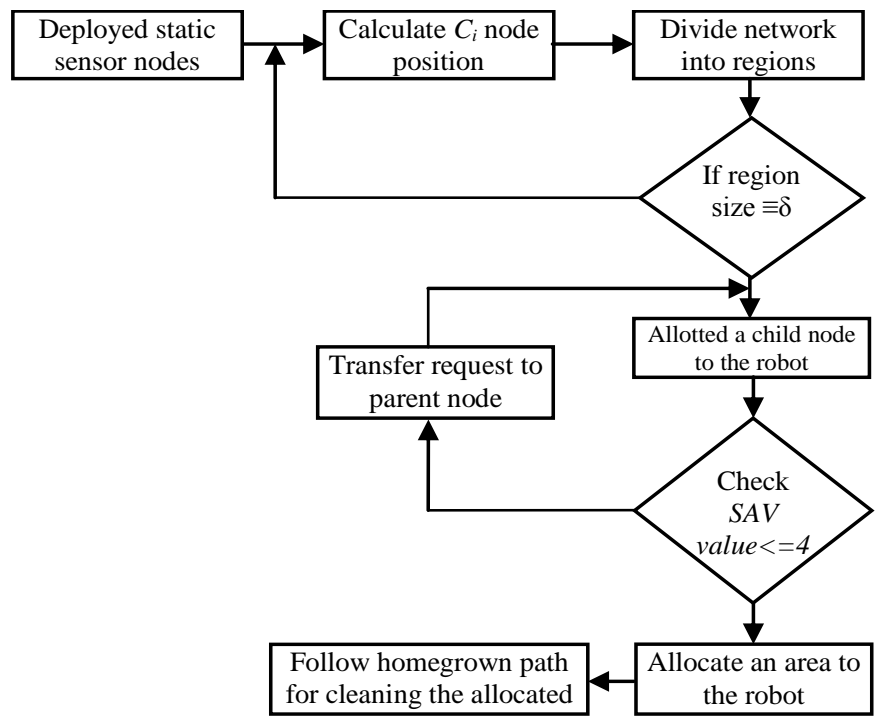

Fig. 4. Area allocation scheme.

\section{EXPERIMENTS}

A testbed of the proposed area allocation scheme has been created for demonstration. The testbed setup is shown in Fig. 5. It builds an instance of distributed area allocation and mobile sink movement controls in a typical home or office environment. In this testbed, twenty static sensor nodes are deployed within two rooms, two meeting rooms, and one corridor. The corridor connects two offices, and two rooms. The entire network divides into five regions. Each region is monitored by four sensor nodes. The initial energy of each sensor is 2.5 Joules. 

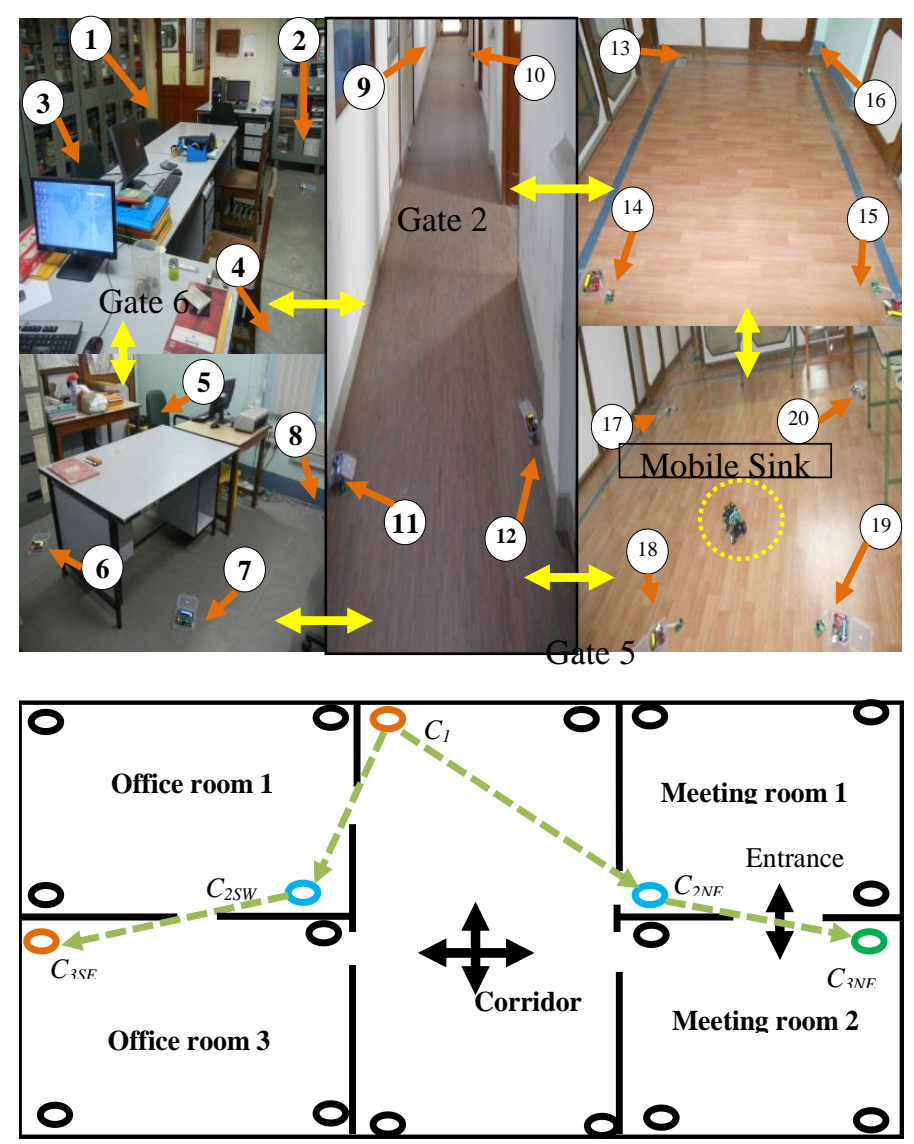

Fig. 5. Cleaning robot assessment in home environment.

\section{A. Data Gathering Tour Length}

In the mobile sink based data gathering process, the mobile sink visits each region or sub-network according to the quad tree information and collects data from each sub network or room. Sensor nodes are deployed in $100 \mathrm{~m}$ $\times 100 \mathrm{~m}$ home area. A robot starts each data gathering tour from the common place and return to the same place after each tour. The tour length of the robot is computed by summing the internal trip mobility path length, the external trip path length and the homegrown mobility path length.

Fig. 6 shows that total data gathering tour length within the home area. It is easy to observe that the data gathering tour length of the proposed scheme is less than the random robot mobility scheme. This is due to effective assessment of the data gathering area through the quad tree management scheme. Quad tree based robot allocation and maneuvering process reduces the overlapping cleaning areas of a cleaning tour that then reduces the tour length for the mobile robot. 


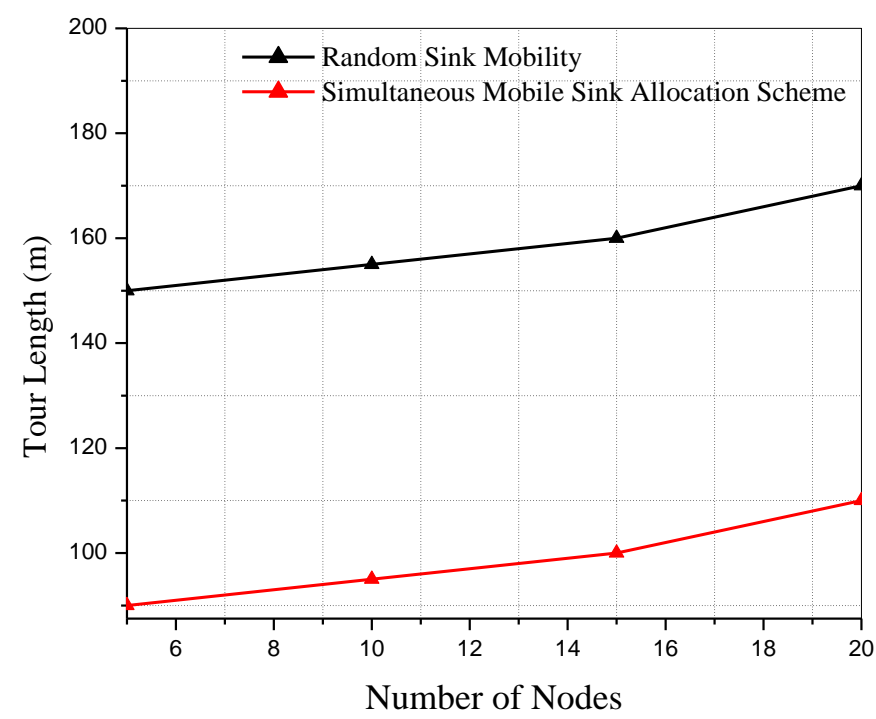

Fig. 6. Single hop data gathering tour length with one mobile sink.

\section{B. Energy Dissipation}

The lifetime of the robotic vacuum cleaner control network is evaluated through the energy dissipation rate of the static sensor nodes. Energy dissipation of the network after the end of each data gathering tour is calculated by the summation of the residual energy of static nodes.

Fig. 7 shows the energy dissipation of the network with one mobile sink. It is easy to observe that the energy dissipation of the proposed scheme is less than the random sink mobility scheme. In the random mobility scheme, the mobile sink can visit a particular static sensor several times in a data gathering tour and collect data from the same node several times. However, some sensor nodes are uncovered by the mobile sink in several data gathering tours. Therefore, the overall energy dissipation rate in the random sink mobility based data gathering scheme is very high compared to the proposed scheme. On the other hand, in the proposed scheme mobile sink reaches each sensor node and directly collects data from the static sensor nodes. Therefore, energy dissipation rate in the proposed scheme is low compared to random sink mobility schemes.

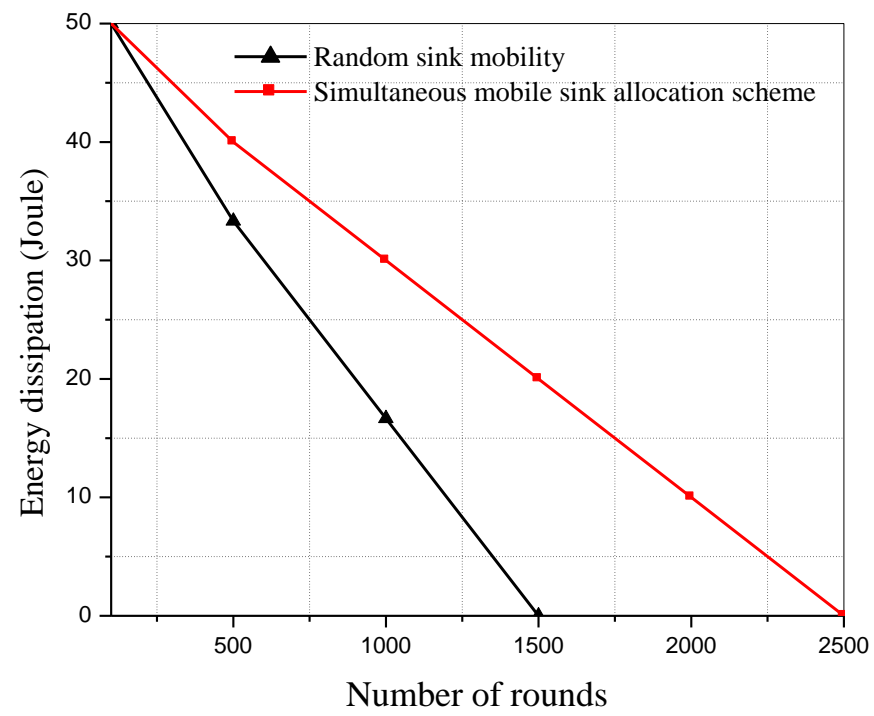

Fig. 7. Energy dissipation of network in mobile sink based data gathering. 


\section{Robotic Cleaner Performance}

Here, the performance of the robotic cleaner with the mobile sink based data gathering network is evaluated in terms of tour length, rate of cleaning area allocation failure rate, cleaning area assessment rate and total assessment delay as presented in TABLE I. It can be seen that as the number of static sensor nodes increases then the cleaning area allocation rate is also increased and the cleaning area allocation failure rate decreases. This is due to the fact that if the node numbers are increased within the network, the BS receives more update messages from the network in a reduced time interval and it therefore increases the allocation rate of the robotic cleaner. However, TABLE 1 also shows the variation of the cleaning area allocation delay time for different numbers of sensor nodes. It is seen that if number of sensor nodes is increased, then the total allocation delay time also decreases due to a decrease in the cleaning area allocation failure rate.

TABLE I

Robotic VACuUm Cleaner Performance

\begin{tabular}{ccccc}
\hline \hline $\begin{array}{c}\text { Number of } \\
\text { deployed } \\
\text { sensors }\end{array}$ & $\begin{array}{c}\text { Tour } \\
\text { length } \\
(\mathbf{m})\end{array}$ & $\begin{array}{c}\text { Allocation } \\
\text { Failure } \\
\text { Rate (\%) }\end{array}$ & $\begin{array}{c}\text { Cleaning } \\
\text { Area } \\
\text { Allocation } \\
\text { Rate (\%) }\end{array}$ & $\begin{array}{c}\text { Total } \\
\text { Allocation } \\
\text { Delay (s) }\end{array}$ \\
\hline 4 & 50.08 & 35 & 84 & 42 \\
8 & 70.07 & 31 & 88 & 38 \\
12 & 80.76 & 28 & 90 & 34 \\
16 & 90.23 & 25 & 93 & 30 \\
20 & 98.06 & 20 & 96 & 25 \\
\hline
\end{tabular}

TABLE II shows the comparison results between the random sink mobility based robotic vacuum cleaner management scheme and quad tree based robotic vacuum cleaner management scheme. In the random sink mobility based management scheme, the overlapping area is $50.5 \%$ higher compared to the quad tree based sink allocation scheme. Also, in the random sink mobility scheme the data gathering latency and robotic vacuum cleaner allocation latency are twice that of the quad tree based scheme. The quad tree based effective sink allocation scheme reduces robotic vacuum cleaner allocation scheme through sequential data collection.

TABLE II

DATA GATHERING LATENCY IN OFFICE/HOME ENVIRONMENT

\begin{tabular}{cccc}
\hline \hline Sink movement strategy & $\begin{array}{c}\text { Overlapping } \\
\text { area }\left(\mathbf{m}^{\mathbf{2}}\right)\end{array}$ & $\begin{array}{c}\text { Mobile sink } \\
\text { data } \\
\text { gathering } \\
\text { latency }(\mathbf{s})\end{array}$ & $\begin{array}{c}\text { Robotic } \\
\text { vacuum } \\
\text { cleaner control } \\
\text { latency }(\mathbf{s})\end{array}$ \\
\hline $\begin{array}{c}\text { Random sink mobility } \\
\text { Quad tree based sink mobility }\end{array}$ & 60 & 50 & 35 \\
\hline
\end{tabular}

\section{CONClusion}

This paper has proposed a simultaneous mobile sink allocation scheme ideal for typical home environments with a clear application for consumer based robotic vacuum cleaners. In the proposed scheme, mobile sinks collect data from the deployed static sensors and transmits it to the BS. According to the static sensors information, the BS automatically navigates the mobile sink to a target location in the home environment. The proposed scheme minimizes the cleaning time through optimal mobility of mobile sink in the home or office environment. The cleaning robot control is simulated with real-world sensing information before being physically tested in a typical home/office scenario. The proposed scheme can effectively assign a cleaning area to a robotic vacuum cleaner without the need for any direct human control. 


\section{REFERENCES}

[1] S. Kim, J.-Y. Sim, and S. Yang, "Vision-based cleaning area control for cleaning robots," IEEE Trans. Consum. Electron. vol. 58, no. 2, pp. 685-690, May 2012.

[2] J. Wang, Y. Yin, J. Zhang, S. Lee, and R. S. Sherratt, "Mobility based energy efficient and multi-sink algorithms for consumer home networks," IEEE Trans. Consum. Electron. vol. 59, no. 1, pp. 77-84, Feb. 2013.

[3] P. Chanak, I. Banerjee, J. Wang, and R. S. Sherratt, "Obstacle avoidance routing scheme through optimal sink movement for home monitoring and mobile robotic consumer devices," IEEE Trans. on Consum. Electron., vol. 60, no. 4, pp. 596-606, Nov. 2014

[4] I. A. Zualkernan, A. R. Al-Ali, M. A. Jabbar, I. Zabalawi, and A. Wasfy, "InfoPods: Zigbee-based remote information monitoring devices for smart-homes," IEEE Trans. Consum. Electron., vol. 55, no. 3, pp. 1221 1226, Aug. 2009.

[5] N. L. Doh, C. Kim, and W. K. Chung, "A practical path planner for the robotic vacuum cleaner in rectilinear environments," IEEE Trans. Consum. Electron., vol. 53, no. 2, pp. 519-527, Nov. 2007.

[6] J. S. Oh, Y. H. Choi, J. B. Park, and Y. F. Zheng, "Complete coverage navigation of cleaning robots using triangular-cell-based map," IEEE Trans. Ind. Electron., vol.51, No. 3, pp. 718 - 726, Jun. 2004.

[7] M.-C. Kang, K.-S. Kim, D.-K. Noh, J.-W. Han, and S.-J. Ko, "A robust obstacle detection method for robotic vacuum cleaners," IEEE Trans. Consum. Electron., vol. 60, no. 4, pp. 587 - 595, Nov. 2014.

[8] C.-H. Kuo, H.-C. Chou, and S.-Y. Tasi, "Pneumatic sensor: a complete coverage improvement approach for robotic cleaners," IEEE Trans. Instrum. Meas., vol. 60, no. 4, pp. 1237-1256, Apr. 2011.

[9] Y.-W. Bai and M.-F. Hsueh, "Using an adaptive iterative learning algorithm for planning of the path of an autonomous robotic vacuum cleaner," in Proc. IEEE Global Conf. Consum. Electron., Tokyo, Japan, pp. 401-405, Oct. 2012.

[10] I. Banerjee, P. Chanak, H. Rahaman, and T. Samanta, "Effective fault detection and routing scheme for wireless sensor networks," Computers \& Electrical Engineering, Elsevier, vol. 40, no. 2, pp. 291-306, Feb. 2014.

[11] S. Saeedi, L. Paull, M. Trentini, and H. Li, "Neural network-based multiple robot simultaneous localization and mapping," IEEE Trans. Neural Netw., vol. 22, no. 12, pp. 2376-2387, Dec. 2011.

[12] D. T. Lee and R.L. Drysdale, "Generalization of Voronoi diagrams in the plane," SIAM J. Computing, vol. 10, no. 1, pp. 73-87, Feb. 1981.

[13] H. Choset and J. Burdick, "Sensor based motion planning: the hierarchical generalized Voronoi graph," SAGE Int. J. Robotics Research, vol. 19, no. 2, pp. 96-125, Feb. 2000.

[14] R. Geraerts and M. Overmars, "Creating small roadmaps for solving motion planning problems," in Proc. IEEE Int. Conf. Methods and Models in Automation and Robotics, Miedzyzdroje, Poland, pp. 531-536, 2005

[15] R. Sugthara and R. K. Gupta, "Improving the data delivery latency in sensor networks with controlled mobility," in Proc. 4th IEEE Int. Conf. Distributed Computing in Sensor Systems, Santorini Island, Greece pp. 386-399, Jun. 2008. 


\section{BIOGRAPHIES}

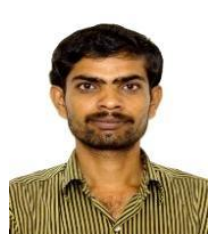

Prasenjit Chanak (S'12) received the B.Tech degree in information Technology for the Institute of Engineering and Technology (IET), Jaunpur, India in 2007 followed by the M.Tech degree in information technology from Bengal Engineering and Science University (BESU), Shibpur, West Bengal, India in 2011 and is currently working toward the Ph.D. degree in Information Technology at Indian Institute of Engineering Science and Technology (IIEST), Shibpur, West Bengal, India.

He is the recipient of a senior research fellowship (SRF), CSIR, Govt. of India. His research interests are in the areas of wireless ad hoc and sensor networks and wireless and mobile systems.

Indrajit Banerjee (M'11) received the Bachelor degree in Mechanical Engineering from Institute of Engineers, India, the Masters in Information Technology from Bengal Engineering and Science University, and the PhD. in Information Technology from Indian Institute of Engineering Science and Technology (IIEST), Shibpur, India.

$\mathrm{He}$ is currently an assistant professor in the information technology department at the Indian Institute of Engineering Science and Technology (IIEST), Shibpur, India. His current interests are cellular automata, wireless ad hoc and sensor network, embedded systems and pervasive computing.

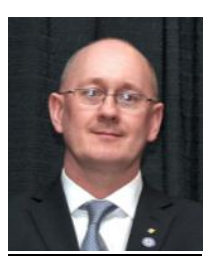

R. Simon Sherratt (M'97-SM'02-F'12) received the B.Eng. degree in Electronic Systems and Control Engineering from Sheffield City Polytechnic, UK in 1992, M.Sc. in Data Telecommunications in 1994 and Ph.D. in video signal processing in 1996 from the University of Salford, UK.

In 1996, he was appointed as a Lecturer in Electronic Engineering at the University of Reading where he is now a Professor of Consumer Electronics and Head of Wireless and Computing research. His research topic is signal processing in consumer electronic devices.

Eur Ing Professor Sherratt was an IEEE Consumer Electronics Society Vice President (08-09) and a serving AdCom member $(03-08,10-15)$. He received the IEEE Chester Sall Memorial $1^{\text {st }}$ Place Award in 2006 and is now the Editor-in-Chief of the IEEE TRANSACTIONS ON CONSUMER ELECTRONICS. 